\title{
Outcome of cats with diarrhea and Tritrichomonas foetus infection
}

\author{
Derek M. Foster, DVM; Jody L. Gookin, DVM, PhD, DACVIM; Matthew F. Poore, BS; \\ Marty E. Stebbins, DVM, PhD, DACVPM; Michael G. Levy, PhD
}

Objective-To determine the long-term outcome of cats infected with Tritrichomonas foetus and identify treatment and management strategies influencing resolution of infection or associated diarrhea.

\section{Design-Prospective study.}

Sample Population-26 cats with $T$ foetus-associated diarrhea at least 22 months prior to the study.

Procedure-A standardized survey regarding clinical course and management was administered to owners of cats with $T$ foetus infection and associated diarrhea. Fecal samples were obtained from each cat; the presence of $T$ foetus was assessed via microscopic examination of smears, culture in commercial media, and polymerase chain reaction amplification of $T$ foetus rDNA involving species-specific primers.

Results-Survey responses were obtained from owners of all 26 cats. Twenty-three cats had complete resolution of diarrhea a median of 9 months after onset. Analysis of fecal samples obtained from 22 cats revealed persistent $T$ foetus infection in 12, with a median of 39 months after resolution of diarrhea. History of implementation of a dietary change, treatment with paromomycin, or higher numbers of cats in the household was associated with significantly longer duration of time to resolution of diarrhea.

Conclusions and Clinical Relevance-Results suggested chronic $T$ foetus-associated diarrhea in most cats is likely to resolve spontaneously within 2 years of onset. Chronic infection with $T$ foetus (without clinical signs) after resolution of diarrhea appears to be common. Although often temporarily effective in decreasing severity of diarrhea, attempts to treat cats with $T$ foetus infection may result in prolongation of time to resolution of diarrhea. (J Am Vet Med Assoc 2004;225:888-892)

$T$ ritrichomonas foetus is a single-celled, flagellated protozoan that colonizes the colon of domestic cats resulting in chronic large-bowel diarrhea. ${ }^{1}$ The infection is prevalent among cats in shelters and purebred show cats, and its presence is significantly associated with his-

From the Department of Molecular Biomedical Sciences (Foster, Gookin) and Population Health and Pathobiology (Poore, Stebbins, Levy), College of Veterinary Medicine, North Carolina State University, Raleigh, NC 27606. Dr. Foster's present address is the Department of Clinical Sciences, College of Veterinary Medicine and Biomedical Sciences, Colorado State University, Fort Collins, CO 80523.

Funded by a grant from Fort Dodge Animal Health. Dr. Foster was supported by a grant from Merck-Merial.

Presented in abstract form at the 21st Annual Forum of the American College of Veterinary Internal Medicine, Charlotte, NC June 2003.

Address correspondence to Dr. Gookin. tory of diarrhea within the cattery. ${ }^{2}$ Trophozoites of $T$ foetus can be difficult to distinguish from Giardia sp except that the former possess a distinct undulating membrane, do not form cysts, and are refractory to treatment with antiprotozoal drugs, such as metronidazole and fenbendazole. ${ }^{a}$ In cats, infection with $T$ foetus is diagnosed by microscopic examination of a fecal smear, protozoal culture of feces by use of a commercially available system, 3,b or extraction of DNA from feces and amplification of $T$ foetus rDNA via a polymerase chain reaction (PCR) technique involving species-specific primers. ${ }^{5}$

At present, there are no effective treatments for eradication of $T$ foetus infection or management of the associated diarrhea in cats. The diarrhea appears to be prolonged, and veterinary practitioners have no information regarding the outcome of diarrhea or course of infection with which to advise owners of their cats' prognoses. In our experience, attempts to treat affected cats with $T$ foetus-associated diarrhea in continued high-density housing results in prolongation of clinical signs. Numerous cats are euthanatized as a result of their uncertain future health and potential to infect other cats. The purpose of the study reported here was to determine the long-term outcome of cats with $T$ foetus infection and identify treatment and management strategies that influence resolution or prolongation of infection or associated diarrhea.

\section{Materials and Methods}

Study population and survey-Ten owners of 26 cats for which a diagnosis of diarrhea and $T$ foetus infection had been made a minimum of 22 months prior to the initiation of the study were contacted. Infection with $T$ foetus had been definitively diagnosed in all cats on the basis of findings of a microscopic examination of a direct fecal smear, microbial culture of feces by use of modified Diamond's medium ${ }^{c}$ or a commercially available culture system, ${ }^{3, \mathrm{~b}}$ or PCR amplification of $T$ foetus rDNA involving species-specific primers. ${ }^{4}$

A standardized survey consisting of 14 questions requiring discrete answers was conducted by telephone with each owner. Survey questions were designed to determine the onset, severity, and progression of diarrhea associated with $T$ foetus infection in cats. Owners were asked to score the consistency of their cats' feces on a scale of 1 to 5 (a score of 1 represented watery feces and 5 represented feces that were dry and firm). Frequency of abnormal stools was categorized as greater than once per day, once per day, a few times per week, once per week, or less than once per week. Owners were asked to identify any attempts made at medical, dietary, or environmental management of $T$ foetus infection and resultant effects on severity of diarrhea. Information regarding the number of other cats in the household and their $T$ foetus infection status was also collected.

Sample collection and analysis-At the completion of the survey, each owner was requested to provide a fecal sam- 
ple from their infected cats. For cats that resided in the local area, feces were collected by the authors by use of a plastic loop inserted rectally, whereas fecal samples obtained from the remaining cats were shipped to our facility by mail. For sample collection from the latter cats, owners were mailed 2 kits/cat with each kit containing 1 pair of latex examination gloves, 1 commercially available culture system, ${ }^{b}$ a plastic vial containing $5 \mathrm{~mL}$ of $70 \%$ ethanol, and 2 wooden applicator sticks. Owners were instructed to isolate the affected cat until it defecated; they were to collect a sample of feces that had been passed $<8$ hours previously and had minimal cat litter embedded in it. Each kit contained written instructions to inoculate the culture system ${ }^{\mathrm{b}}$ with approximately $0.025 \mathrm{~g}$ of feces (about the size of a grain of rice) and collect enough feces to fill the volume remaining in the plastic vial (approximately $1 \mathrm{~mL}$ ). Owners were instructed to mail the samples to the authors in an addressed, stamped envelope that was provided.

Analysis of fecal samples for $T$ foetus included microscopic examination of a fecal smear, culture of feces in the commercially available culture system ${ }^{\mathrm{b}}$ or modified Diamond's medium, ${ }^{\mathrm{c}}$ and single-tube nested PCR assay for $T$ foetus rDNA involving species-specific primers. Fecal smears were prepared by touching a wooden applicator stick to the fecal sample and mixing it with a drop of physiologic saline $(0.9 \% \mathrm{NaCl})$ solution on a microscope slide. A coverslip was placed on the slide prior to examination by use of a light microscope (200X magnification). A commercially available culture system $^{\mathrm{b}}$ was inoculated with $0.025 \mathrm{~g}$ of feces, incubated in an upright position at room temperature $\left(25^{\circ} \mathrm{C}\right)$, and examined via light microscopy (200X magnification) daily for 2 weeks, as described. ${ }^{3}$ For culture in modified Diamond's medium, ${ }^{c} 0.1 \mathrm{~g}$ of feces was suspended in $10 \mathrm{~mL}$ of sterile phosphate-buffered saline (PBS; $0.9 \% \mathrm{NaCl}$ ) solution. A $100-\mu \mathrm{L}$ aliquot of this solution was inoculated into a culture tube containing $10 \mathrm{~mL}$ of modified Diamond's medium ${ }^{\mathrm{c}}$ fortified with antimicrobials $\left(10^{6} \mathrm{U}\right.$ of penicillin/L, $15 \mathrm{~g}$ of streptomycin/L, and $2 \mathrm{mg}$ of amphotericin $\mathrm{B} / \mathrm{L}$ ) and incubated at $37^{\circ} \mathrm{C}$. Cultures were examined once daily for 1 week. All light microscopic examinations were performed by personnel (DMF, JLG, and MFP) experienced in the differentiation of $T$ foetus and Giardia sp trophozoites.

By use of a commercial kit ${ }^{\mathrm{d}}$ under conditions optimized for elimination of PCR inhibitors, DNA was extracted from 200-mg samples of feces. A single-tube nested PCR assay was performed by use of primers designed to specifically amplify a partial ITS1 and 5.8S rRNA gene sequence of $T$ foetus. Primer sequences and reaction conditions have been previously published. ${ }^{4}$ Amplicons were detected via UV illumination after electrophoresis of $10 \mu \mathrm{L}$ of the reaction solution in a $1.5 \%$ agarose gel containing ethidium bromide. To avoid PCR contamination, the DNA extractions, reaction preparation, thermal cycling, electrophoresis, and detection of amplicons were performed in separate laboratories. During DNA extractions, a tube containing PBS solution was processed in parallel with other study samples to detect any genomic DNA contamination. As negative controls, tubes containing either $5 \mu \mathrm{L}$ of sterile water or $5 \mu \mathrm{L}$ of the product obtained from processing sterile PBS solution as if DNA were being extracted were included in each PCR experiment to detect any amplicon or genomic DNA contamination, respectively. As a positive control, 1 tube containing $0.5 \mu \mathrm{L}$ of purified $T$ foetus genomic DNA and $4.5 \mu \mathrm{L}$ of sterile water instead of sample DNA was included in each PCR experiment. The PCR was repeated as many as 10 times on DNA extracted from feces of each cat to increase sensitivity for detection of $T$ foetus.

To examine the time-course of histologic changes in the colon associated with $T$ foetus infection, colonic mucosal biopsy specimens were collected prospectively from a sexual- ly intact female domestic longhair cat that had had chronic large-intestinal diarrhea since 6 weeks of age; specimens were obtained at the time of diagnosis of $T$ foetus infection ( 6 months of age) and 12 and 24 months later. Beginning at 6 months of age, the cat was singly housed, fed a commercially available dry food, ${ }^{\text {e }}$ and received no medical treatment other than use of heartworm prevention medication. Prior to each biopsy procedure, food was withheld for 12 hours and the cat was given 2 warm water enemas. The cat was anesthetized with ketamine $(10 \mathrm{mg} / \mathrm{kg}$ [4.5 mg/lb], IV) and diazepam (1 mg/kg [0.45 mg/lb], IV). Mucosal samples were obtained from the proximal, middle, and distal portions of the colon by use of a suction capsule biopsy instrument. ${ }^{f}$ Specimens were fixed in neutral-buffered $10 \%$ formalin, routinely processed, paraffin embedded, sectioned at a thickness of $5 \mu \mathrm{m}$, and stained with $\mathrm{H} \& \mathrm{E}$ for examination by use of light microscopy.

Statistical analyses-For categorical data, a Fisher exact test was used to assess for significance. All other variables were assessed by use of a Mann-Whitney or Wilcoxon signed rank test or Spearman rank order correlation where appropriate. A calculated $P$ value of $\leq 0.05$ was considered significant. Statistical analyses were performed by use of a commercially available software package. ${ }^{g}$

\section{Results}

Complete survey responses were obtained from owners of all 26 cats. Many of the owners surveyed had $>1$ cat in the study; 1 owner had 12 infected cats, 5 owners had 2 infected cats each, and the remaining 4 cats were the only infected cats in their households. The time since diagnosis of $T$ foetus infection in the 26 cats ranged from 22 to 66 months (median, 4 years). At the time of diagnosis, cats had abnormal stools either daily (22 cats), weekly (3), or monthly (1). The duration of clinical signs prior to diagnosis of $T$ foetus infection ranged from 0 to 24 months (median, 2.5 months) and began at a median age of 9 months (range, 6 weeks to 6 years). Twenty-five of the cats came from multiple cat households containing 2 to 20 cats. As reported by their owners, 23 of the 26 cats had complete resolution of their clinical signs by a median of 9 months (range, 5 to 24 months) after onset of $T$ foetus-associated diarrhea. Two cats had some improvement in severity of diarrhea beginning after 4 months and 24 months, respectively. Only 1 cat had no change in severity of diarrhea at the time of the survey, which was 24 months after the onset of clinical signs. There was a positive correlation between time from diagnosis to remission of diarrhea and the number of other cats present in the household $(\rho=0.56 ; P=0.003)$.

When owners were requested to provide a current score for their cats' feces on a scale from 1 to 5 , responses ranged from 3 to 5 (median, 3.5). Owners of 25 cats reported a history of resolution or partial improvement of diarrhea, although 12 of these cats had ongoing relapses of diarrhea. Two cats had relapses weekly, 6 cats had relapses monthly, and 4 cats had relapses at $>1$-month intervals. At the times of relapses, assessment of the cats' fecal scores ranged from 1 to 4 (median, 2); these scores were significantly $(P<0.001)$ more severe than the scores reported at the time of the survey. Owners attributed relapses of diarrhea to dietary changes (5 cats), medication unrelated to the $T$ foetus infection (ie, administration of azithromycin to treat a chronic respiratory 
tract infection, medical treatment of lymphoma, and administration of behavior modification agents; 3 cats), travel ( 1 cat), and unknown cause ( 3 cats).

Twenty-four of the 26 cats were treated medically in an attempt to eradicate the $T$ foetus infection; 8 antimicrobial drugs were administered in various combinations. Drugs prescribed and the number of cats that responded to treatment (as indicated by decreased severity of diarrhea) included paromomycin (13/15 cats), metronidazole (1/13), sulfadimethoxine (0/14), fenbendazole (0/13), furazolidone (1/1), enrofloxacin $(0 / 2)$, gentamicin $(0 / 2)$, and cephalexin (0/2). Although of benefit in improving severity of diarrhea in many instances, none of the antimicrobials were effective in sustaining resolution of clinical signs.

Owners of 14 cats attempted to manage their cats' diarrhea by changing the diet. The most commonly chosen foods were a prescription diet formulated for the management of intestinal disorders ${ }^{\mathrm{h}}$ (11 cats), a commercially available diet formulated for cats with sensitive stomachs $^{i}(2)$, and a commercially available turkey and barley diet $t^{j}$ with home-cooked turkey and rice (1). Fecal consistency improved in all 14 cats that underwent a dietary change. However, cats undergoing a dietary trial had significantly $(P=0.004)$ longer time to final resolution of $T$ foetus-associated diarrhea (median, 15 months), compared with cats that did not receive a change in diet (median, 7 months). Dietary supplements were given to 12 cats and included yogurt (3 cats), slippery elm (6), pumpkin (2), and glutamine (1). No dietary supplement was associated with improvement in diarrhea. The owners of 2 cats attempted to manage their cats' diarrhea by bathing them more often and changing their litter box more frequently; however, no improvement in diarrhea was noted in association with those interventions.

Fecal samples were obtained from 22 of the 26 cats included in the survey. The authors were able to collect feces by use of a fecal specimen loop from 15 cats that resided locally; the owners of the remaining 7 cats shipped a voided fecal sample by mail to the authors' laboratory. For each of the 15 cats from which feces were collected by use of a fecal specimen loop, 2 direct fecal smears were prepared; via light microscopy, all smears were negative for $T$ foetus. Fecal specimens obtained from 16 cats were cultured to detect $T$ foetus in commercially available pouches, ${ }^{\mathrm{b}}$ and 1 specimen yielded a positive result. Culture of fecal material in modified Diamond's medium ${ }^{c}$ to detect $T$ foetus was performed on samples obtained from 11 cats, and 1 specimen yielded a positive result. Single-tube nested PCR assay performed on all 22 fecal samples revealed positive results for $T$ foetus in 12 cats, which included the 2 cats with $T$ foetus identified via culture techniques. In those 12 cats, the number of PCR reactions required to detect $T$ foetus rDNA was 1 (8 cats), 2 (1), 3 (2), and 5 (1). All negative-control samples included in each set of extractions and all reaction preparations yielded negative results. Negative results were obtained for all 10 PCR reactions performed on feces from each of the 10 remaining cats. All cats that had infection with $T$ foetus detected at the time of the study were reported by their owners to have had resolution of diarrhea at a median of 39 months prior to testing (range, 16 to 60 months).
There was no significant association between PCR results and duration of clinical signs prior to resolution of diarrhea $(P=0.59)$, history of relapsing diarrhea $(P=$ $0.85)$, prior treatment with paromomycin $(P=0.44)$, implementation of a dietary trial $(P=0.20)$, or number of other cats in the household $(P=0.37)$.

Serial colonic mucosa biopsy specimens were available for histologic examination from a cat that did not receive any medical or dietary intervention following diagnosis of $T$ foetus infection (Figure 1). Examination
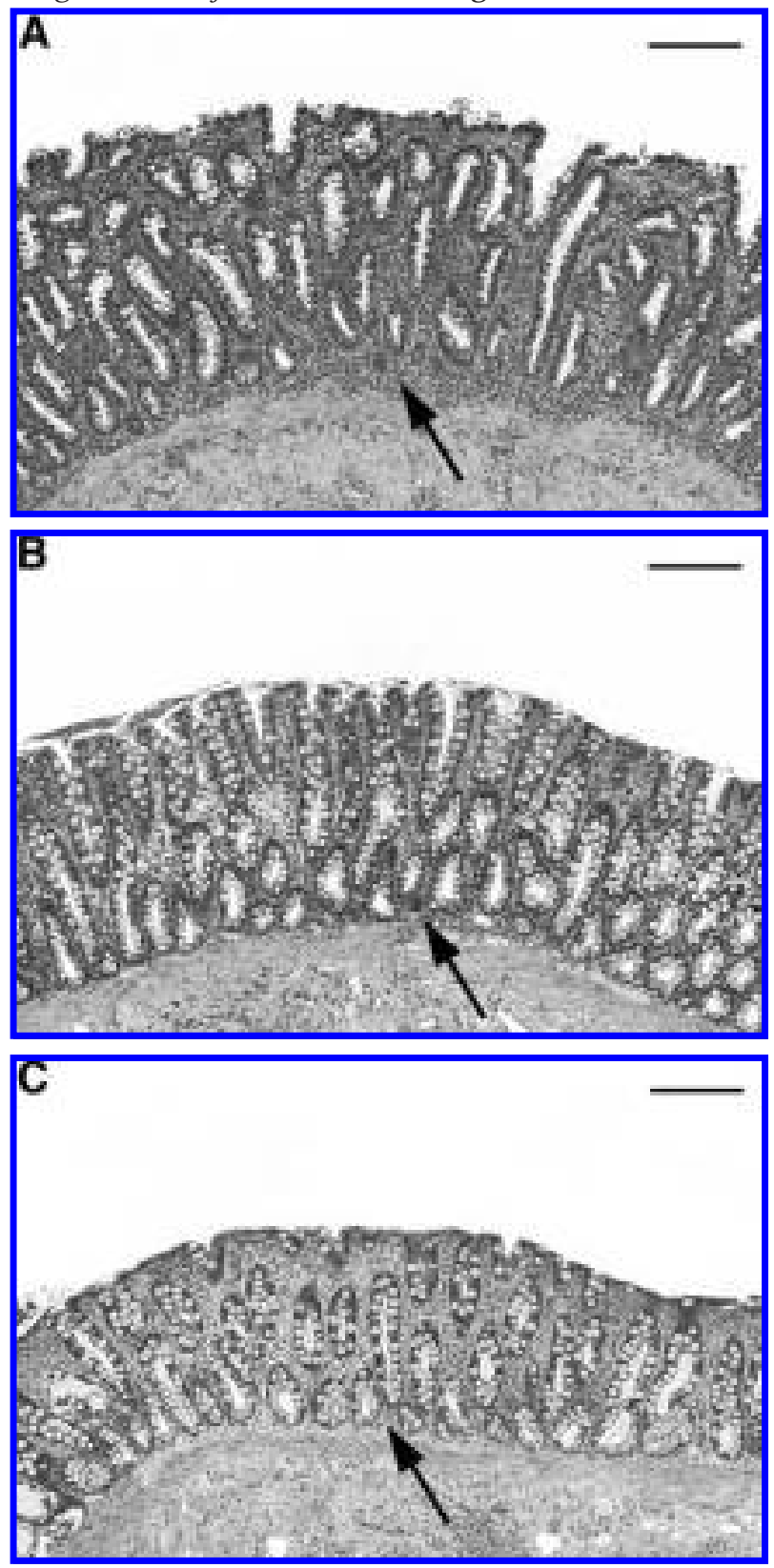

Figure 1-Photomicrographs of sections of colonic mucosa obtained from a cat infected with Tritrichomonas foetus at the time of diagnosis of infection ( 6 months of age; A), 12 months later when diarrhea was beginning to resolve (B), and 24 months after diagnosis when fecal consistency had returned to normal and the result of a polymerase chain reaction assay for $T$ foetus was negative $(\mathrm{C})$. Notice that the degree of lymphocytic-plasmacytic infiltrate in the lamina propria decreased (solid arrows) and disruption of the epithelial surface resolved with time. In all panels, H\&E stain; bar $=150 \mu \mathrm{m}$ 
of biopsy specimens obtained from that cat at the time of diagnosis revealed moderate to severe lymphocyticplasmacytic colitis with multifocal disruption of the surface epithelium. With time, the severity of the cat's diarrhea gradually diminished and histologic examination of biopsy specimens obtained 1 year later revealed mild to moderate lymphocytic-plasmacytic colitis without evidence of epithelial disruption. At 24 months after diagnosis of $T$ foetus infection, the cat's diarrhea had resolved and examination of biopsy specimens revealed no evidence of colonic mucosal inflammation. At the time of the third biopsy procedure, feces from this cat were analyzed via a PCR assay for $T$ foetus infection and the result was negative; at the time of the study of this report (25 months after resolution of diarrhea), the result of a similar PCR assay for $T$ foetus infection was also negative.

\section{Discussion}

In the study of this report, $88 \%$ (23/26) of cats had resolution of their clinical signs within 2 years of onset of $T$ foetus-related diarrhea (median, 9 months). Despite resolution of clinical signs, the owners of many cats reported subsequent infrequent and short-duration relapses of diarrhea. As fecal samples were not obtained at these times, it is unknown if these relapses corresponded with fecal shedding of $T$ foetus. Because we did not survey owners of cats without $T$ foetus infection, the importance of periodic episodes of diarrhea is questionable, especially given the association of such episodes with antimicrobial treatment or dietary change, both of which are common causes for transient diarrhea in cats. There was also no significant relationship between history of relapse of diarrhea and presence of continued infection (determined on the basis of PCR results) at the time of the study.

Results of the culture or PCR assay of feces performed at the time of the study indicated that more than half of the study cats were infected with $T$ foetus despite previous resolution of their clinical signs. The fact that these cats had positive results for $T$ foetus via PCR assay at a median of 39 months after reported resolution of their diarrhea suggests that infections without clinical signs can be prolonged. Persistence of infection in the absence of continued diarrhea may be related to a low number of organisms in these cats or other unidentified factors. Feces from 2 cats yielded positive results via protozoal culture, which is a technique that can detect $1 \times 10^{4}$ organisms/g of feces. ${ }^{3}$ Feces from another 10 cats yielded positive results for $T$ foetus via PCR assay only; the PCR technique used in the study of this report has a practical test sensitivity for detecting as few as 500 organisms/g of feces. ${ }^{4}$ Our observation that between 1 and 5 PCR reactions were required to amplify $T$ foetus rDNA from each fecal sample obtained suggests that the number of organisms harbored by these 10 cats was quite low. It is possible that PCR testing of multiple fecal samples from each cat in the study would reveal additional cats that remained infected with $T$ foetus. We believe that laboratory contamination with $T$ foetus genomic DNA or PCR amplicons in our study was unlikely, as false positive results were not obtained from negative-control samples included in each set of extractions and all reaction preparations. The importance of latent infections to spread of $T$ foetus or later development of secondary sequelae is unknown. There were no common features among $T$ foetus-infected versus non-infected cats with respect to duration of diarrhea prior to resolution, history of periodic relapse of diarrhea, number of other cats in each household, or attempts at antimicrobial or dietary management of infection.

Numerous antimicrobial drugs and several specially formulated diets were administered to the cats of the study of this report in an attempt to eradicate or manage the diarrhea resulting from $T$ foetus infection. Of note was our observation that a change in diet or treatment with paromomycin, an aminoglycoside that is reportedly poorly absorbed from the gastrointestinal tract after oral administration, was associated with improvement in severity of diarrhea in several cats. As determined by prior studies, ${ }^{5, a}$ paromomycin is incapable of killing $T$ foetus in vitro or in vivo. In the study of this report, there was no association between either a change in diet or treatment with paromomycin and the cats' status regarding $T$ foetus infection. It is plausible that these treatments caused an alteration in colonic microbial populations that resulted in temporary improvement in diarrhea. Anecdotally, we have observed that cats treated with antimicrobial drugs or undergoing a change in diet have delayed resolution or exacerbation of their diarrhea. ${ }^{6}$ Cats treated with paromomycin or undergoing a change in diet in the present study had significantly longer time to eventual resolution of $T$ foetus-associated diarrhea. These findings suggest that imposed alterations in colonic microflora or other unidentified effects of these treatments do not result in long-term benefit. In addition, acute renal failure, deafness, and blindness have been attributed to absorption of paromomycin across damaged intestinal epithelium in cats with $T$ foetus infection. ${ }^{\top}$

In our study, there was a positive correlation between the number of cats in each household and the duration of the interval to resolution of $T$ foetus-associated diarrhea. This could be attributed to increased stress or reinfection of cats under dense housing conditions. It is also possible that these results are biased by our inclusion in the study of infected cats that were residing in the same household as other infected cats.

Results of the study of this report have suggested that most cats diagnosed with chronic diarrhea resulting from $T$ foetus infection will have apparent spontaneous resolution of diarrhea within 2 years of onset. In a single cat that was housed alone and not undergoing any form of treatment, spontaneous resolution of diarrhea and clearance of $T$ foetus infection (as determined via PCR assay) were accompanied by complete remission of colonic inflammation. Among the cats of our study, chronic $T$ foetus infection without clinical signs after resolution of diarrhea was common. Although temporarily effective in decreasing severity of diarrhea in many cats, attempts to treat $T$ foetus infection often resulted in prolongation of time to resolution of diarrhea.

${ }^{a}$ Levy MG, Gookin JL, Poore MF, et al. Intestinal trichomonosis in cats: pathology, diagnosis and susceptibility to antiprotozoal drugs (abstr), in Proceedings. Joint Meet Am Soc Parasitol Soc Protozool 2000;108. 
${ }^{b}$ In Pouch TF, Biomed Diagnostics Inc, White City, Ore. 'Modified Diamond's medium, Remel, Lenexa, Kan.

'QIAamp DNA stool mini kit, Qiagen, Valencia, Calif.

${ }^{e}$ Hill's feline maintenance light diet, Hill's Pet Nutrition Inc, Topeka, Kan.

${ }^{\mathrm{f}}$ Model $4.7 \mathrm{~mm} \times 120 \mathrm{~cm}$, Quinton Instrument Co, Seattle, Wash.

${ }^{8}$ Analyse-It, version 1.63, Analyse-It Software Ltd, Leeds, UK.

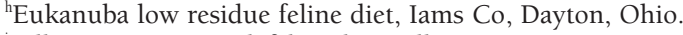

${ }^{i} H$ Hill's sensitive stomach feline diet, Hill's Pet Nutrition Inc, Topeka, Kan.

${ }^{j}$ Purina turkey and barley diet, Nestle-Purina, Vevey, Switzerland.

\section{References}

1. Levy MG, Gookin JL, Poore MF, et al. Tritrichomonas foetus and not Pentatrichomonas hominis is the etiologic agent of feline trichomonal diarrhea. IParasitol 2003;89:99-104.

2. Gookin JL, Stebbins ME, Hunt E, et al. Prevalence of and risk factors for Tritrichomonas foetus and Giardia infection. $\underline{\text { LClin Microbiol }}$ 2004:42:2707-2710.

3. Gookin JL, Foster DM, Poore MF, et al. Use of a commercially available culture system for diagnosis of Tritrichomonas foetus infection in cats. I Am Vet Med Assoc 2003; 222:1376-1379.

4. Gookin JL, Birkenheuer AJ, Breitschwerdt EB, et al. Singletube nested PCR assay for detection of Tritrichomonas foetus in feline feces. LClin Microbiol 2002;40:4126-4130.

5. Gookin JL, Breitschwerdt EB, Levy MG, et al. Diarrhea associated with trichomonosis in cats. LAm Vet Med Assoc 1999;215:1450-1454.

6. Gookin JL, Levy MG. Trichomonosis in cats: recognition and resilience, in Proceedings. 19th Annu Am Coll Vet Intern Med Forum 2001;753-755.

7. Gookin JL, Riviere JE, Gilger BC, et al. Acute renal failure in four cats treated with paromomycin. IAm Vet Med Assoc 1999;215: 1821-1823. 